\title{
"EosFit-Pinc: A simple GUI for host-inclusion elastic thermobarometry"-Reply to Zhong et al.
}

\author{
Ross J. Angel ${ }^{1, *}$, Mattia L. Mazzucchelli ${ }^{2}$, Matteo Alvaro $^{2,} \uparrow$, and Fabrizio Nestola ${ }^{3,}$ \\ ${ }^{1}$ IGG-CNR, Via G. Gradenigo 6, Padova, 35131, Italy \\ ${ }^{2}$ Department of Earth and Environmental Sciences, University of Pavia, Via A. Ferrata, 1, Pavia 27100, Italy \\ ${ }^{3}$ Department of Geosciences, University of Padova, Via G. Gradenigo 6, Padova 35131, Italy
}

\begin{abstract}
We provide a further algebraic proof that the lines of entrapment conditions for inclusions calculated with the formula of Guiraud and Powell (2006) are not thermodynamic isomekes and therefore do not represent exactly lines of possible entrapment conditions.
\end{abstract}

Keywords: Isomekes, inclusions, EosFit-Pinc, thermodynamics

\section{INTRODUCTION}

Zhong et al. (2020) argue that the solution for entrapment conditions of inclusions presented by Guiraud and Powell (2006) is as equally valid as that proposed by Angel et al. (2017). In addition they propose a third calculation route based on the logarithmic definition of strain and a change in reference conditions. For the example that Zhong et al. (2020) show in their Figure 1, all three methods yield calculated entrapment pressures at metamorphic temperatures that differ by a small amount that is larger than numerical rounding error in the calculations. However, if the three solutions yield three different sets of entrapment pressures for a given temperature and the same input parameters, then the three different results cannot all be simultaneously thermodynamically correct. The essential question is therefore: which one is thermodynamically correct?

The thermodynamic concept behind the determination of conditions of entrapment of inclusions within host minerals is the isomeke (Rosenfeld and Chase 1961; Adams et al. 1975). The isomeke is a thermodynamically defined line along which the fractional volume changes of two phases (host and inclusion) remain equal but non-zero. Therefore, if two $P-T$ points, $a$ and $b$, lie on the same isomeke of the host $h$ and inclusion $i$ their volumes at these two points are related exactly by:

$$
\frac{V_{\mathrm{i}}\left(P_{\mathrm{a}} T_{\mathrm{a}}\right)}{V_{\mathrm{i}}\left(P_{\mathrm{b}} T_{\mathrm{b}}\right)}=\frac{V_{\mathrm{h}}\left(P_{\mathrm{a}} T_{\mathrm{a}}\right)}{V_{\mathrm{h}}\left(P_{\mathrm{b}} T_{\mathrm{b}}\right)} .
$$

The consequence, for host-inclusion calculations, is that an inclusion that perfectly fits the hole it occupies in a host crystal at the time of entrapment will continue to do so at all $P-T$ conditions along the isomeke line through entrapment conditions, without developing additional stresses, regardless of the slope of the isomeke. The inclusion pressure therefore remains equal to the external pressure along this entrapment isomeke (e.g., Angel et al. 2015). And inclusions trapped at different points along the same host-inclusion isomeke will exhibit the same residual pressure as one another when examined at room conditions (or any other

\footnotetext{
* E-mail: rossjohnangel@gmail.com

$\uparrow$ ORCID 0000-0002-6975-3241

† ORCID 0000-0002-4875-5125

Open access: Article available to all readers online. This article is CC-BY
}

$P$-T point away from the entrapment isomeke). The consequence is that unique entrapment conditions cannot be inferred from the measurement of the residual pressure in an inclusion alone under the assumption that the phases are isotropic; only the entrapment isomeke (a line in $P-T$ space) can be inferred.

Therefore, one can determine which model or models for inclusion entrapment pressures are valid by testing whether or not they predict entrapment conditions that all lie on a single isomeke from a single value of $P_{\text {inc. }}$. We first apply this condition to the equation of Guiraud and Powell (2006) to prove that it does not represent an isomeke. Then we confirm the algebraic analysis by simply calculating from the individual EoS of the phases the ratios in Equation 1 along the predicted line of entrapment.

\section{Algebraic proof}

The equation given by Guiraud and Powell (2006) that relates the $P_{\text {trap }}$ and $T_{\text {trap }}$ to $P_{\text {inc }}$ at room temperature when the host pressure is zero (and vice versa), is:

$$
\frac{3 P_{\text {inc }}}{4 G_{\mathrm{h}}}=\frac{V_{\mathrm{i}}\left(P_{\text {inc }} T_{\text {room }}\right)}{V_{\mathrm{i}}\left(P_{\text {trap }} T_{\text {trap }}\right)}-\frac{V_{\mathrm{h}}\left(P_{\text {room }} T_{\text {room }}\right)}{V_{\mathrm{h}}\left(P_{\text {trap }} T_{\text {trap }}\right)} .
$$

We now multiply the first term on the right by the ratio of volumes of the inclusion at one point on the isomeke at room temperature

$$
\frac{V_{\mathrm{i}}\left(P_{\text {foot }} T_{\text {end }}\right)}{V_{\mathrm{i}}\left(P_{\text {foot }} T_{\text {end }}\right)}=1,
$$

and the second by the same volume ratio (also of unity) for the host. Then the Guiraud and Powell (2006) Equation 2 becomes:

$$
\left[\frac{3 P_{\text {inc }}}{4 G_{\mathrm{h}}}\right]=\left[\frac{V_{\mathrm{i}}\left(P_{\text {foot }} T_{\text {end }}\right)}{V_{\mathrm{i}}\left(P_{\text {trap }} T_{\text {trap }}\right)}\right]\left[\frac{V_{\mathrm{i}}\left(P_{\text {inc }} T_{\text {room }}\right)}{V_{\mathrm{i}}\left(P_{\text {foot }} T_{\text {end }}\right)}\right]-\left[\frac{V_{\mathrm{h}}\left(P_{\text {foot }} T_{\text {end }}\right)}{V_{\mathrm{h}}\left(P_{\text {trap }} T_{\text {trap }}\right)}\right]\left[\frac{V_{\mathrm{h}}\left(P_{\text {inc }} T_{\text {room }}\right)}{V_{\mathrm{h}}\left(P_{\text {foot }} T_{\text {end }}\right)}\right]
$$$$
[A]=[B] \quad[C]-[D] \quad[E]
$$

The second line in Equation 3 is just labels that we have assigned to the corresponding terms in the full equation to make the following explanation clearer. The condition that $P_{\text {foot }}, T_{\text {end }}$ and $P_{\text {trap }}, T_{\text {trap }}$ both lie on the same isomeke is, from Equation 1, the condition $[B]=[D]$.

If we have measured $P_{\mathrm{inc}}$ at room temperature and we know $G_{\mathrm{h}}$, 
we can solve Equation 3 for $P_{\text {trap }}=P_{\text {foot }}$ and $T_{\text {trap }}=T_{\text {end }}$ to define one point on the entrapment isomeke, at $P_{\text {foot }}, T_{\text {end. }}$. At these conditions, Equation 3 is algebraically identical to the solution given in Angel et al. (2017), and the terms $[A]$, $[C]$, and $[E]$ are now fixed. With these terms fixed as defining one point on the entrapment isomeke, there are no other solutions to Equation 3 unless $[B] \neq[D]$, which implies a violation of the isomeke condition of Equation 1 which in turn shows that Guiraud and Powell (2006) equation cannot represent an isomeke. The same conclusion can be reached another way: if $[B]=[D]$ then we can write Equation 3 as $[A]=[B]([C]-[E])$. Since $[A],[C]$, and $[E]$ are fixed, this tells us there is only one value of $[B]$ that simultaneously satisfies both the isomeke condition and the Guiraud and Powell (2006) equation; again a demonstration that the Guiraud and Powell (2006) equation cannot represent an isomeke.

Figure 1c of Zhong et al. (2020) shows as an example the calculated entrapment conditions for a quartz inclusion in almandine garnet with a residual inclusion pressure of $0.6 \mathrm{GPa}$ when the host garnet is at room conditions. Table la shows entrapment pressures calculated with the Guiraud and Powell (2006) and Angel et al. (2017) models, at room $T$ and $750{ }^{\circ} \mathrm{C}$. The table shows that the fractional volume changes of the host and inclusion change along the entrapment line calculated with the Guiraud and Powell (2006) model, confirming that it is not an isomeke. The differences are larger when the contrast between the bulk moduli of the host and inclusion is smaller, for example for zircon in pyrope (Table 1b). While the differences in these two examples may not be geologically significant, the errors for other host-inclusion pairs cannot be predicted without explicit calculation.

The proof can also be performed with the same conclusion but with more complex working for $P-T$ points other than $P_{\text {foot }}, T_{\text {end. }}$. This shows that we previously erred in stating that the relaxation has to be calculated isothermally; this should have been obvious from the fact that the solution of $P_{\text {inc }}$ was presented in Angel et al. (2017) in terms of force balance at the final conditions. Therefore, we agree with Zhong et al. (2020) that this is not the cause of the difference between the Guiraud and Powell (2006) and Angel et al. (2017) solutions that they show in their Figure 1. However, we also want to note that, contrary to the statements in Zhong et al. (2020), there is no restriction in this analysis or that of Angel et al. (2017) to the final conditions being at room conditions.

\section{Conclusion}

We have proved that the lines of entrapment conditions calculated by the method of Guiraud and Powell (2006) are not thermodynamically correct because they are not exactly isomekes. The source of this difference was shown in Equations A6 and A9 of Angel et al. (2017) and is the factor that we here call

$$
\left[\frac{V_{\mathrm{i}}\left(P_{\text {foot }} T_{\text {end }}\right)}{V_{\mathrm{i}}\left(P_{\text {trap }} T_{\text {trap }}\right)}\right]=\left[\frac{V_{\mathrm{h}}\left(P_{\text {foot }} T_{\text {end }}\right)}{V_{\mathrm{h}}\left(P_{\text {trap }} T_{\text {trap }}\right)}\right],
$$

the fractional volume change of the two phases along the entrapment isomeke. When this factor is close to unity, the solution of Guiraud and Powell (2006) is a close approximation to the thermodynamically correct solution. The same is true for the logarithmic basis proposed as an alternative by Zhong et al. (2020). Factors that further reduce the accuracy of the Guiraud and Powell (2006) approximation include hosts with small shear
TABLE 1a. Quartz in garnet for $P_{\text {inc }}=0.6 \mathrm{GPa}$ at $25^{\circ} \mathrm{C}$

\begin{tabular}{lccccccc}
\hline$T_{\text {trap }}$ & $P_{\text {trap }}($ exact $)$ & $P_{\text {trap }}(\mathrm{G} \& \mathrm{P})$ & \multicolumn{2}{c}{$V N_{\text {foot }}$ (exact) } & & \multicolumn{2}{c}{$V N_{\text {foot }}(\mathrm{G} \& \mathrm{P})$} \\
& $(\mathrm{GPa})$ & $(\mathrm{GPa})$ & host $=[\mathrm{D}]$ & inc $=[\mathrm{B}]$ & & host $=[\mathrm{D}]$ & inc $=[\mathrm{B}]$ \\
\hline 25 & 1.048 & $1.050^{\mathrm{a}}$ & 1.00000 & 1.00000 & & 1.00000 & 1.00000 \\
750 & 1.871 & 1.876 & 1.01175 & 1.01175 & & 1.01173 & 1.01168 \\
\hline
\end{tabular}

TABLE 1b. Zircon in Garnet for $P_{\text {inc }}=0.25 \mathrm{GPa}$ at $25^{\circ} \mathrm{C}$

\begin{tabular}{|c|c|c|c|c|c|c|}
\hline \multirow{2}{*}{$\begin{array}{l}T_{\text {trap }} \\
\left({ }^{\circ} \mathrm{C}\right)\end{array}$} & \multirow{2}{*}{$\begin{array}{c}P_{\text {trap }} \text { (exact) } \\
(\mathrm{GPa})\end{array}$} & \multirow{2}{*}{$\begin{array}{c}P_{\text {trap }}(\mathrm{G} \& \mathrm{P}) \\
(\mathrm{GPa})\end{array}$} & \multicolumn{2}{|c|}{$V / V_{\text {foot }}($ exact $)$} & \multicolumn{2}{|c|}{$V / N_{\text {foot }}(G \& P)$} \\
\hline & & & host $=[D]$ & $\overline{\text { inc }}=[\mathrm{B}]$ & host $=[\mathrm{D}]$ & inc $=[B]$ \\
\hline 25 & -1.751 & $-1.753^{a}$ & 1.00000 & 1.0 & 00000 & 000 \\
\hline 800 & 4.778 & 4.801 & 0.98122 & 0.98122 & 0.98108 & 0.98112 \\
\hline
\end{tabular}

Notes: All calculations performed with EosFit7c (Angel et al. 2014). All of the EoS used in these examples can be downloaded as .eos files from www.rossangel.net. a The values of $P_{\text {trap }}$ at $25^{\circ} \mathrm{C}$ are $P_{\text {foot }}$ and differ from the exact solution because they do not include the non-linearity of the $P-V$ relationship in the host and inclusion (Angel et al. 2017).

moduli (Eq. 2) and systems that have a high contrast in values of the pressure derivatives of their bulk moduli (i.e., $K^{\prime}$ ) leading to strongly curved isomekes, such as often occur in mixed-phase inclusions (e.g., Musiyachenko et al. 2020). Furthermore, the differences in the calculated entrapment conditions between the approach of Guiraud and Powell (2006) and the thermodynamic isomekes are further magnified when unique entrapment conditions are being inferred from the anisotropy of strains of the trapped inclusions (e.g., Alvaro et al. 2020). On the other hand, the approach of Angel et al. (2017) is thermodynamically correct for all cases because it calculates points on the entrapment isomeke from $P_{\text {foot }} T_{\text {end }}$ by explicitly enforcing the isomeke condition. We therefore recommend that all host-inclusion calculations are based on the thermodynamically correct basis of the isomeke, regardless of the value of the final $P_{\text {trap. }}$

\section{FUNDING}

Our current research on host-inclusion systems is supported by the European Research Council under the European Union's Horizon 2020 research and innovation program grant agreement 714936 TRUE DEPTHS to Matteo Alvaro at the University of Pavia. The work reported in the paper under discussion was supported by ERC starting grant 307322 to Fabrizio Nestola, and by the MIUR-SIR grant "MILE DEEp" (RBSI140351) to Matteo Alvaro.

\section{REFERENCES CITED}

Adams, H.G., Cohen, L.H., and Rosenfeld, J.L. (1975) Solid inclusion piezothermometry I: comparison dilatometry. American Mineralogist, 60, 574-583.

Alvaro, M., Mazzucchelli, M.L., Angel, R.J., Murri, M., Campomenosi, N., Scambelluri, M., Nestola, F., Korsakov, A.V., Tomilenko, A.A., Marone, F., and Morana, M. (2020) Fossil subduction recorded by quartz from the coesite stability field. Geology, 48, 24-28.

Angel, R.J., Gonzalez-Platas, J., and Alvaro, M. (2014) EosFit7c and a Fortran module (library) for equation of state calculations. Zeitschrift für Kristallographie, 229, 405-419.

Angel, R.J., Nimis, P., Mazzucchelli, M.L., Alvaro, M., and Nestola, F. (2015) How large are departures from lithostatic pressure? Constraints from host-inclusion elasticity. Journal of Metamorphic Geology, 33, 801-803.

Angel, R.J., Mazzucchelli, M.L., Alvaro, M., and Nestola, F. (2017) EosFit-Pinc: A simple GUI for host-inclusion elastic thermobarometry. American Mineralogist, 102, 1957-1960.

Guiraud, M., and Powell, R. (2006) $P-V-T$ relationships and mineral equilibria in inclusions in minerals. Earth and Planetary Science Letters, 244, 683-694.

Musiyachenko, K.A., Murri, M., Prencipe, M., Angel, R.J., and Alvaro, M. (2020) A Grüneisen tensor for rutile and its application to host-inclusion systems. American Mineralogist, in review.

Rosenfeld, J.L., and Chase, A.B. (1961) Pressure and temperature of crystallization from elastic effects around solid inclusion minerals? American Journal of Science, 259, 519-541.

Zhong, X., Dabrowski, M., Powell, R., and Jamtveit, B. (2020) "EosFit-Pinc: A simple GUI for host-inclusion elastic thermobarometry" by Angel et al. (2017) —Discussion. American Mineralogist, 105, 1585-1586.

MANUSCRIPT RECEIVED MAY 18, 2020

MANUSCRIPT ACCEPTED JUNE 10, 2020

MANUSCRIPT HANDLED BY DON BAKER 